\title{
Extracranial Metastasis of IDH-1 Wild Type Glioblastomas
}

\author{
Emre BILGIN ${ }^{1}$, Ali Ihsan OKTEN ${ }^{1}$, Berna BOZKURT DUMAN², Gokhan CAVUS ${ }^{1}$, Vedat ACIK¹, \\ Ismail ISTEMEN ${ }^{1}$, Suleyman ALTINTAS ${ }^{3}$
}

\begin{abstract}
${ }^{1}$ Adana City Training and Research Hospital, Department of Neurosurgery, Adana, Turkey
${ }^{2}$ Adana City Training and Research Hospital, Department of Medical Oncology, Adana, Turkey

${ }^{3}$ Adana City Training and Research Hospital, Department of Pathology, Adana, Turkey
\end{abstract}

Corresponding author: Emre BILGIN dremreblgn@gmail.com

\section{ABSTRACT}

AIM: To identify the effects of different immunohistochemical features of glioblastomas with spinal metastases based on the metastatic spread and survival rate.

MATERIAL and METHODS: A total of 214 patients who were diagnosed with and operated for brain tumor in our clinic between 2007 and 2018 and pathologically diagnosed with glioblastoma were retrospectively evaluated. Among them, 141 medical records were reviewed, and 23 of them underwent spinal magnetic rezonance imaging postoperatively due to various complaints.

RESULTS: All patients with glioblastoma with spinal metastases had negative isocitrate dehydrogenase 1 in the immunohistochemical examination. The incidence of spinal metastasis is 1.91\%. The median Ki-67 index is 30 (range, 4-90; median Ki-67 index: $30+/-$ 18.5). Isocitrate dehydrogenase mutation is wild in $55 \%$, mutant in $33 \%$, and not otherwise specified in $12 \%$. Four patients with spinal metastasis has wild-type IDH with mean Ki-67 index of 60, and one of them was a woman (25\%) and the remaining three were men (\%75), with mean age of 32 years.

CONCLUSION: Gliomas with high immunohistochemical proliferation indexes and wild-type isocitrate dehydrogenase with poor prognostic features based on the new classification tended to metastasize to the spine in the early disease stage; therefore, early spinal scanning and radiation therapy might extend the life expectancy. High Ki-67 index and the presence of wild-type isocitrate dehydrogenase may be the predictive factors for spinal screening.

KEYWORDS: Extracranial metastasis, Glioblastoma multiforme, Wild type, Spinal

\section{INTRODUCTION}

$\mathrm{G}$ lioblastoma (GBM) is the most common primary malignant tumor in the central nervous system (21), with the worst 5-year survival rate among human neoplasms (8). It has a very low rate of spinal metastasis (0.4-2.0\%), and patients diagnosed with spinal metastases die within 2 or 3 months (24). However, spinal metastases are usually asymptomatic. Low back pain, leg pain, sensory loss, or urinary incontinence are symptoms suggesting spinal metastasis in patients with cranial GBM (21). Spinal metastasis can be assessed through routine spinal magnetic resonance imag- ing (MRI) scan after the diagnosis. Therefore, the number of patients with primary intracranial GBM who are diagnosed with spinal metastases is increasing nowadays $(7,17)$. Extracranial metastasis of GBM occurs in the white matter through leptomeningeal or hematogenous routes $(6,13)$. This study investigated symptomatic metastases detected in spinal MRI performed during the preliminary diagnostic period of patients with cerebral GBM or during follow-ups. Therefore, this study aimed to identify the effects of detecting the metastasis and the survival rate based on similarities and differences of immunohistochemical parameters in patients with symptomatic spinal metastasis.

\footnotetext{
Emre BILGIN $\quad$ (D) : 0000-0002-2394-1503

Ali Ihsan OKTEN $\quad$ (D) : 0000-0003-0292-201X

Berna BOZKURT DUMAN (D) : 0000-0003-0295-6295

Gokhan CAVUS (10): 0000-0001-7078-2501

Vedat ACIK (D) : 0000-0002-0371-5883

Ismail ISTEMEN (1) : 0000-0002-2341-4818

Suleyman ALTINTAS (10) : 0000-0002-4468-4120
} 


\section{MATERIAL and METHODS}

A total of 214 patients operated in our clinic with the diagnosis of brain tumor between 2007 and 2018 and pathologically diagnosed with GBM were retrospectively evaluated. This study was approved by the local ethic committee of the Adana City Education and Research Hospital (Date: 21.11.2018; number: 319). Only 141 medical files were reviewed. Patients were excluded from the study in accordance to the exclusion and inclusion criteria. Only patients with pathology confirmed glioblastoma multiforme (GBM) were included. All cranial and spinal MRIs were examined retrospectively. Four patients with metastases are among the 23 patients who underwent MRI.

\section{Statistical Analysis}

The Wolfram Mathematica and E-view programs were used for statistical analyses.

\section{RESULTS}

\section{Patient Demographics}

Medical records of 141 patients with GBM, consisting of 81 (57\%) men and 60 (43\%) women, were reviewed, with an average of 55.2 (range, 5-88) years and average followup period of 11.4 (range, 2-18) months. Among them, only four were diagnosed with symptomatic spinal metastases. The immunohistochemical examination of all patients with GBM with spinal metastases had negative isocitrate dehydrogenase-1 (IDH-1), and their cerebrospinal fluid (CSF) cytology was reportedly positive for malignancy, except for one patient. The incidence of symptomatic spinal metastasis is $1.91 \%$ in our series. The median Ki-67 index is 30 (range, 4-90; median ki67 index, 30+/-18.5). IDH mutation is wildtype in $55 \%$, mutant in $33 \%$, and NOS in $12 \%$ of patients. Four patients with spinal metastasis had wild-type IDH, with the mean Ki-67 index of 60 (range, 40-90), and one of them was a woman $(25 \%)$ and the remaining patients were men (75\%), with mean age of 32 years (Table I).

The first patient was a 46-year-old man who visited our clinic for consultations due to complaints of headache, nausea, and sensory loss at the right side of his body in the last 10 days. An obscure weakness was detected at the right side of his body during the neurological examination. In his phasecontrast MRI, a solid mass was detected in the left temporal lobe showing a heterogeneous appearance (Figure 1A). Left temporal craniotomy and gross total excision of the mass were performed. The surgically resected mass was later examined immunohistochemically and its histopathologic diagnosis was GBM [World Health Organization (WHO) Grade IV] (Figure 1B) with glial fibrillary acidic protein (GFAB) +, Vimentin +, S-100 + , P53 +, and $\mathrm{Ki}-67$ proliferation index of $40 \%$. IDH-1 was immunohistochemically negative. The patient was followed up at the oncology department postoperatively and was administered 60 Gy radiotherapy for 6 weeks concurrently with chemotherapy and adjuvant oral temozolomide treatment. At 16 months postoperatively, the patient was reexamined with phase-contrast cerebral MRI and spinal MRI due to complaints of headache, nausea, paraparesis, bladder/ bowel incontinence, and weakness in left side of his body. Cerebral MRI revealed a local recurrence of tetraparesis in left temporoparietal junction which is more significant in the left side, and spinal MRI revealed a mass spreading from $\mathrm{C} 2$ to T2 that extended to the whole spinal cord (Figure 1C). Then, 8-12 mg of dexamethasone were administered daily for acute treatment for up to 2 weeks intravenously and subsequently orally in tapering doses. Radiotherapy of 2.5 Gy was also administered four times a week for 5 weeks, with a total of 50 Gy doses administered over the spinal area. At 17 months after the first cranial surgery, the patient died due to systemic complications (Table II).

Table I: Radiological Features, Clinical Presentation and Demographic Data of Patients Spinal Metastasis

\begin{tabular}{|c|c|c|c|c|c|c|c|c|c|c|c|}
\hline & Age & Gender & $\begin{array}{c}\text { Spinal location of } \\
\text { Metastasis }\end{array}$ & $\begin{array}{c}\text { Back } \\
\text { pain }\end{array}$ & $\begin{array}{c}\text { Radicular } \\
\text { distrubution }\end{array}$ & Paraparesis & $\begin{array}{l}\text { Autonomic } \\
\text { dysfunction }\end{array}$ & $\begin{array}{l}\text { Sensory } \\
\text { level }\end{array}$ & $\begin{array}{c}\mathrm{Ki}-67 \\
\%\end{array}$ & $\begin{array}{c}\text { IDH-I } \\
\text { Mutation }\end{array}$ & $\begin{array}{c}\text { CSF } \\
\text { cytology }\end{array}$ \\
\hline Patient 1 & 46 & M & $\mathrm{C} 2-\mathrm{T} 2$ & No & No & Yes & Yes & Yes & 40 & Wild type & Yes \\
\hline Patient 2 & 24 & $\mathrm{~F}$ & $\mathrm{C} 2-\mathrm{C} 4$ and T2 & Yes & No & Yes & Yes & Yes & 90 & Wild type & Yes \\
\hline Patient 3 & 26 & M & C3-C5 & No & No & No & Yes & Yes & 40 & Wild type & No \\
\hline Patient 4 & 32 & M & Th12-L1 & Yes & Yes & Yes & Yes & No & 70 & Wild type & Yes \\
\hline
\end{tabular}

Table II: Approaches for Treatment of Spinal Metastasis

\begin{tabular}{|c|c|c|c|c|}
\hline & Patient 1 & Patient 2 & Patient 3 & Patient 4 \\
\hline Antineoplastic therapy & Yes & Yes & Yes & Yes \\
\hline Steroids & Yes & Yes & Yes & Yes \\
\hline Spinal surgery & Not done & Not done & Not done & Not done \\
\hline
\end{tabular}



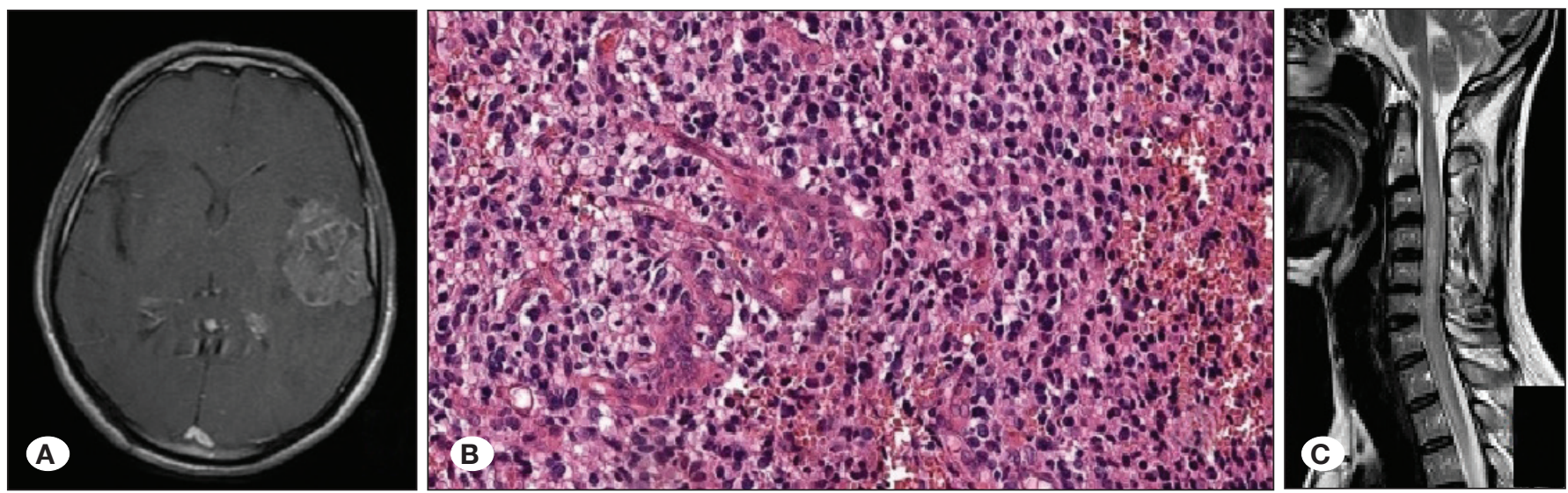

Figure 1: A) Preoperative phase-contrasted axial MRI tumoural mass showing irregular peripheral heterogeneous contrast enhancement which is located in temporal-parietal lobe of left cerebral hemisphere. B) 10450X20 Microscopic image of cerebral biopsy material in hematoxylin eosin staining. C) T2W sagittal cervical MRI shows the space occupying hyperintense expansile lesion in spinal cord at the level of C2-T2 vertebral levels.

The second patient was a 24-year-old woman who visited the clinic with complaints of headache, nausea, vomiting, and facial drooping. Neurological examination revealed facial paralysis on the right side of the face, bilateral vertical gaze limitation, and mild tetraparesis that affected the right side. Cerebral MRI showed a cystic mass lesion with solid components showing heterogeneous contrast enhancement that pushes the brain stem inside the $4^{\text {th }}$ ventricle (Figure 2A). Gross total mass excision was performed on the ventricular mass using the median suboccipital craniotomy method. The surgically resected lesion was later examined immunohistochemically, and its histopathologic diagnosis was GBM (WHO grade IV) (Figure 2B) with GFAB +, Vimentin +, S-100 +, P53 +, and $\mathrm{Ki}-67$ proliferation index of $90 \%$. The IDH-1 was immunohistochemically negative. The patient was followed up at the postoperative oncology department and administered 60 Gy radiotherapy dose for 4 weeks concurrently with chemotherapy, and adjuvant oral temozolomide treatment was initiated. Repeat cerebral and complete spinal MRI was performed when her complaints of weakness in arms and legs, back pain, paraparesis, and bladder/bowel incontinence during her radiotherapy treatment increased. In her cerebral MRI, multiple focal cystic solid lesions recurred within the $4^{\text {th }}$ ventricle, with newly formed lesions in the left parietal and occipital lobes of the brain showing heterogeneously contrasted appearances. The spinal MRI showed multiple lesions within the spinal canal at the cervical and upper thoracic regions, showing intense contrast enhancement (Figure $2 \mathrm{C}$ ). The patient received from 8 to $12 \mathrm{mg}$ dexamethasone daily for acute treatment for up to 2 weeks intravenously and subsequently orally in tapering dose. Therefore, 2.5 Gy of radiotherapy was administered four times a week for 5 weeks, with a total of 50 Gy radiotherapy dose to the cervical region. The patient died 4 months after the cranial surgery (Table II).

The third patient was a 26-year-old man who visited the clinic due to nausea and vomiting. He was operated in the external center 3 months ago with the diagnosis of GBM. The patient received chemotherapy and radiotherapy after the first surgery. His neurological examination revealed weakness in the upper extremities. The first preoperative MRI was not accessed because the first surgery was performed in the external center. At 3 months postoperatively, contrast-enhanced MRI revealed a recurrent mass in the right frontal lobe (Figure $3 \mathrm{~A})$. The patient complained of bladder/bowel incontinence and weakness in the upper extremities; therefore, contrastenhanced spinal MRI was performed, which showed massenhancing lesions at the C3-C5 level (Figure 3B). Gross total mass excision was performed on the right frontal lobe mass using the frontal craniotomy method. The patient received 8-12 mg of dexamethasone daily for acute treatment for up to 2 weeks intravenously and subsequently orally in tapering doses. The surgically resected tissues were later examined immunohistochemically and its histopathologic diagnosis was GBM (WHO grade IV) with GFAB +, Vimentin +, S-100 + , P53 +, and Ki-67 proliferation index of $40 \%$. The primary radiotherapy area was preserved, and the remaining head was administered 2 Gy dose per day for 15 days with a total of 30 Gy radiotherapy dose to the cranial and cervical regions. Radiotherapy was given with chemotherapy, and adjuvant oral temozolomide treatment was initiated. The patient died 2 months postoperatively (Table II).

The last patient was a 32-year-old man who visited the clinic with complaints of headache and epileptic seizure. His neurological examination was normal. In his phasecontrast MRI, a solid mass was detected in the left frontal lobe showing a heterogeneous appearance (Figure 4A). Left frontal craniotomy and gross total excision of the mass were performed. The surgically resected material was later examined immunohistochemically, and its histopathologic diagnosis was GBM (WHO grade IV) with GFAB +, Vimentin +, S-100 +, $\mathrm{P} 53$ +, and Ki-67 proliferation index of $70 \%$. The IDH-1 was immunohistochemically negative. The patient was followed up at the postoperative oncology department and administered 60 Gy radiotherapy dose for 5 weeks concurrently with chemotherapy, and adjuvant oral temozolomide treatment 

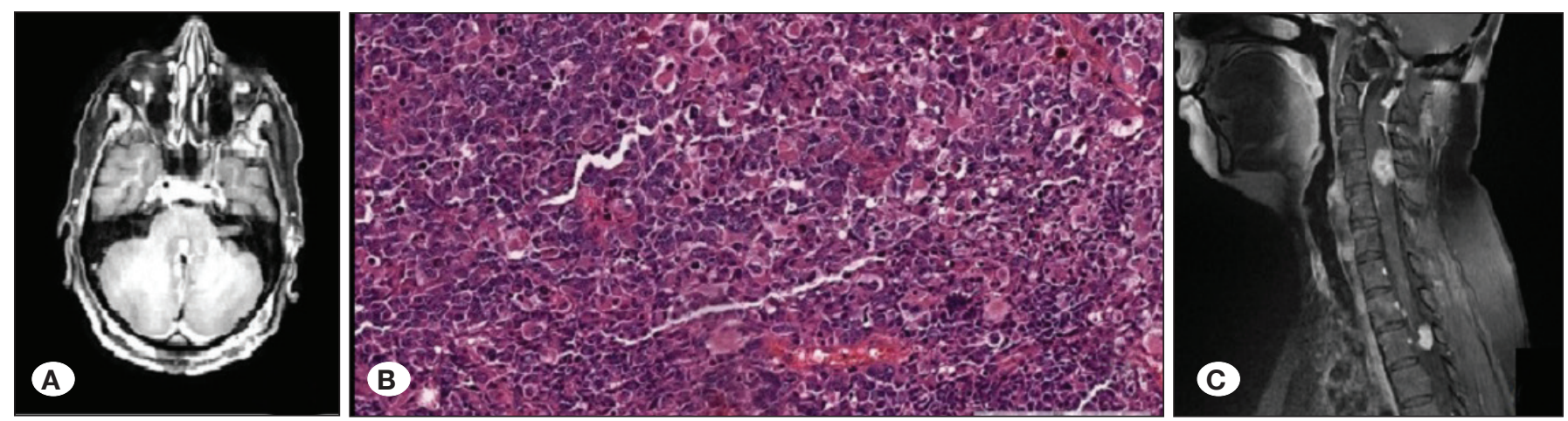

Figure 2: A) Preoperative cerebral axial MRI T2 hyperintense space occupying lesion in posterior fossa midline at the level of middle cerebellar peduncle showing thick peripheral contrast enhancement. B) 10450X20 Microscopic image of cerebral biopsy material in hematoxylin eosin staining. C) Contrast enhanced intramodular lesions in contrasted cervical sagittal MRI. Intramodular mass lesions located in T2 vertebrae level and in C4 vertebral corpus level in spinal cord.
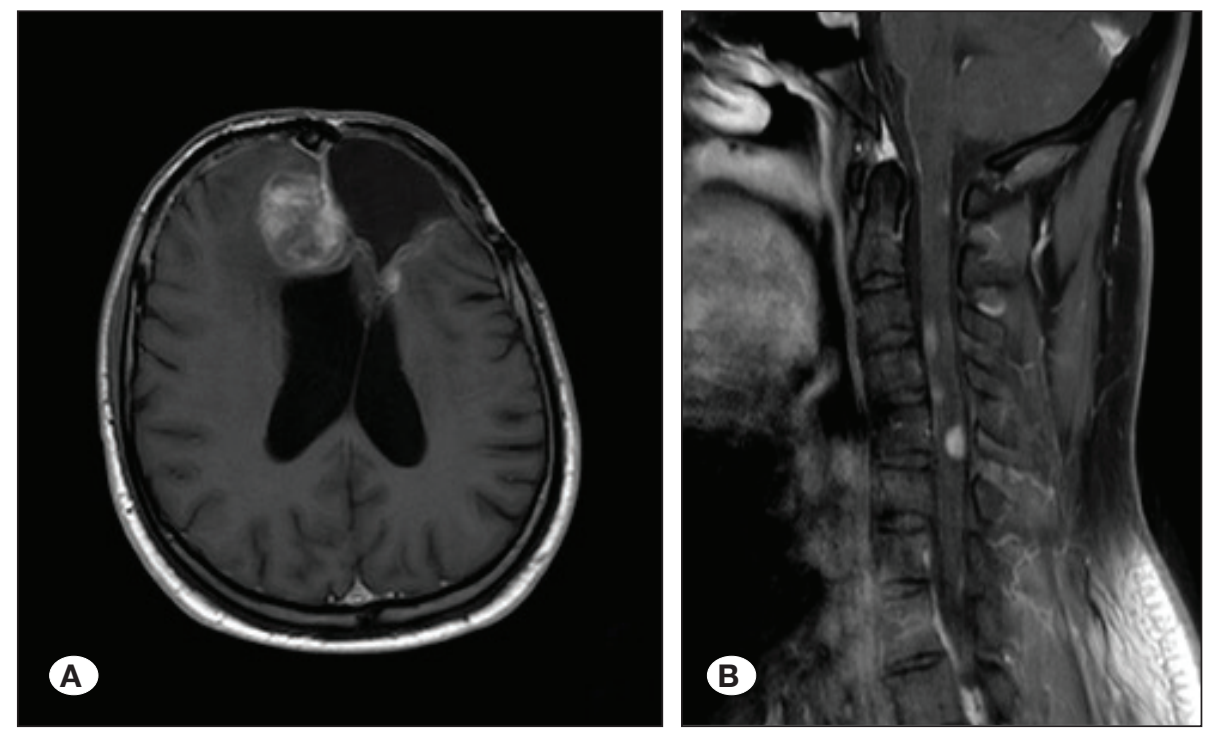

Figure 3: A) Postoperative phasecontrasted cerebral axial MRI nux tumoural mass showing irregular peripheral heterogeneous contrast enhancement which is located in frontal lobe of right cerebral hemisphere. B) T1W sagittal cervical contrast enhanced MRI shows the space occupying hyperintense lesion in spinal cord at the level of C5 vertebral level.

was initiated. At 4 months postoperatively, the patient was reexamined with phase-contrast cerebral MRI and spinal MRI due to complaints of back pain, radicular distribution, paraparesis, headache, nausea, bladder/bowel incontinence, and weakness in both lower extremities. Cerebral MRI revealed a local recurrence in the left frontal lobe, and spinal $M R I$ revealed an image of a mass spreading from T12 to L1 extending to the whole spinal cord (Figure 4B). The patient received 8-12 mg of dexamethasone daily for acute treatment for up to 2 weeks intravenously and subsequently orally in tapering dose. Radiotherapy of 2.5 Gy was administered four times a week for 5 weeks, with a total of 50 Gy over the lumber spinal area. At 3 months after the second cranial surgery, the patient died (Table II).

The overall IDH values among 141 patients data were negative (78 patients), positive (46 patients), and NOS (17 patients). IDH NOS (12\%) had the lease value and negative (55\%) had the maximum value (Figure 5).

Patients complaining of various issues after the surgical operation. The authors observed the clinical and pathological features of 23 patients with GBM. Furthermore, four of 23 (4/23) patients had spinal metastasis with IDH negative (Figure $6)$.

\section{DISCUSSION}

The GBM can spread through CSF due to choroid plexus invasion or cortical surface and subpial metastasis $(6,23)$. Due to anatomic location, symptomatic spinal metastases are commonly observed as leptomeningeal metastases with a rate of $14 \%$ while intramodular metastases rate is $1.1 \%$ in the literature (21). In this retrospective series, the spinal metastatic rate is $1.91 \%$, a little bit higher to that of the world literature. Spinal symptoms occur due to spinal cord conus medullaris compression or untreated cauda equina syndrome. MRI, CSF cytology, and/or a positron emission tomography (PET) scan can be used for diagnosis $(7,17,25)$. Although spinal metastases are reported to spread via the CSF, CSF cytology allows $33 \%$ of patients to be diagnosed for malignancy (10). Ravindran et al. proved that there were cases of GBM spinal metastasis with negative CSF cytology; the mechanism of 

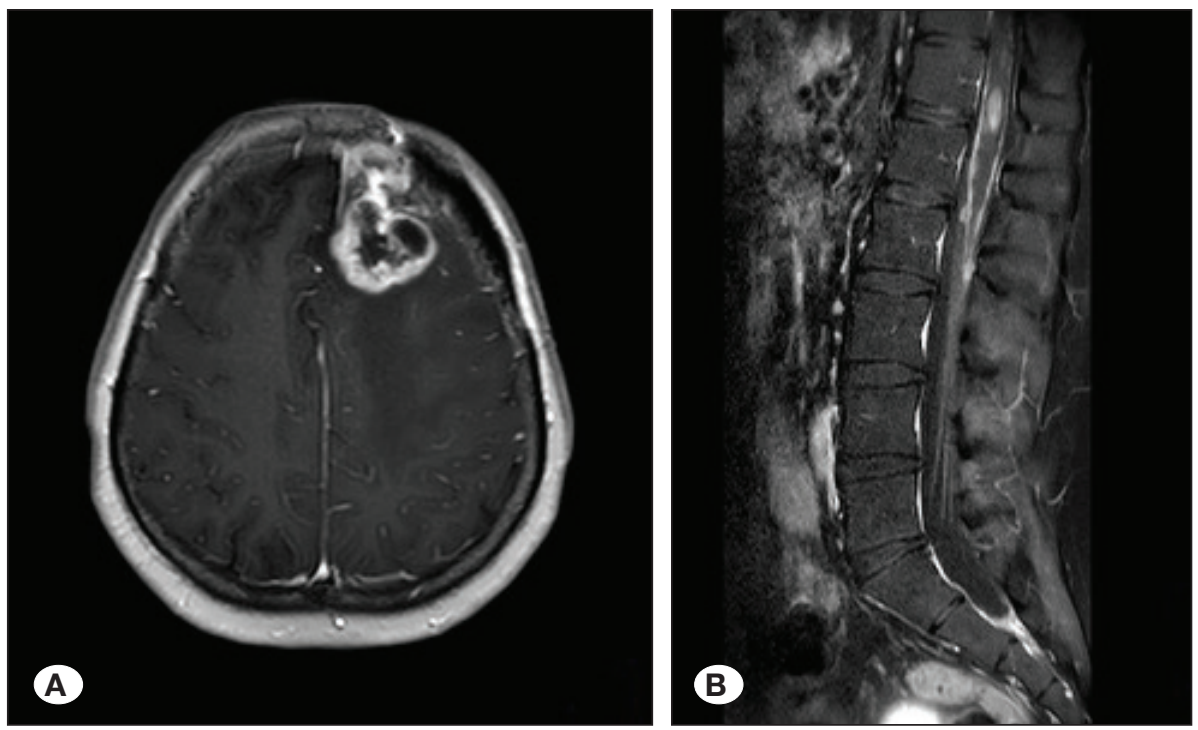

Figure 4: A) Postoperative phasecontrasted cerebral axial MRI nux tumoural mass showing irregular peripheral heterogeneous contrast enhancement which is located in frontal lobe of left cerebral hemisphere. B) T1W sagittal lumbar contrast enhanced MRI shows the space occupying hyperintense lesion in spinal cord at the level of T12-L1 vertebral level.

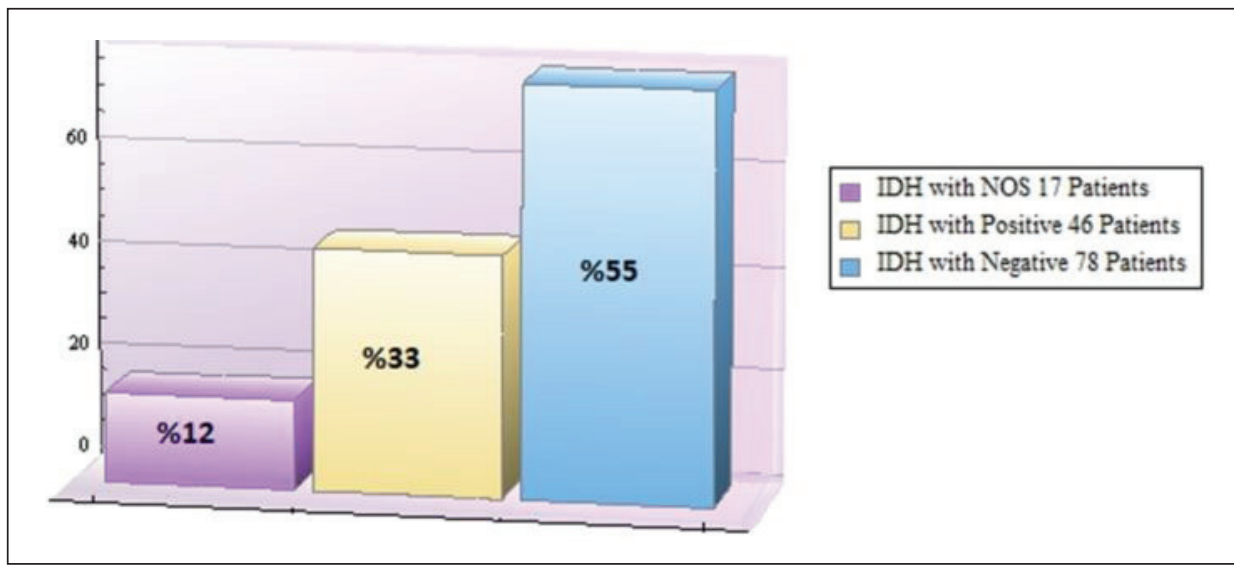

Figure 5: Frequency of negative, positive, NOS values of IDH of 141 patient

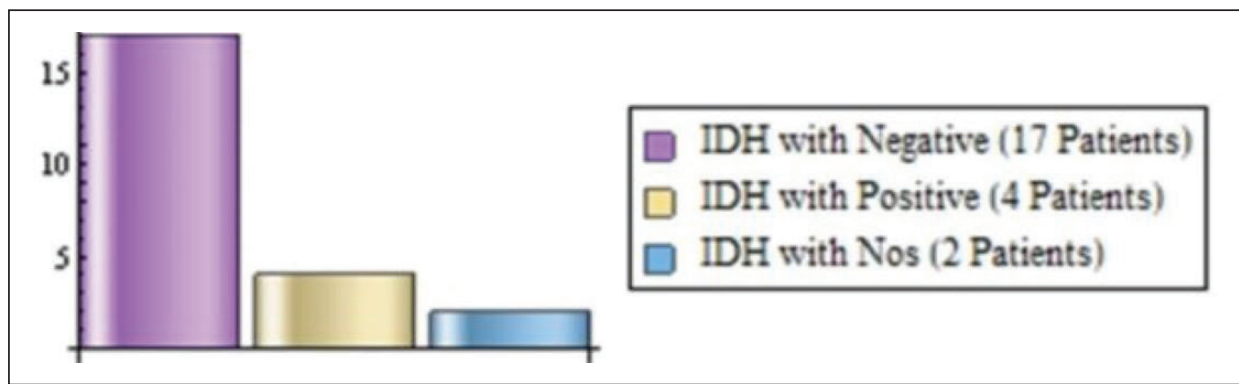

Figure 6: Frequency of among negative, positive, NOS values of IDH of symptomatic 23 patients.

its occurrence remains (12). We used the CSF cytology and spinal MRI to diagnose patients with spinal metastases. Three patients in this case study had a positive CSF cytology for malignancy and died at 2-17 months after their first diagnosis of symptomatic spinal metastases.

Due to the fact that nearly $75 \%$ of all GBM patients die at the early disease stage within 18 months because of intracranial primary tumor, spinal metastases are rare. Symptomatic spinal metastases are observed in young patients and died within 2 to 3 months after the diagnosis $(2,24)$.
High $\mathrm{Ki}-67$ proliferation index is a pathological characteristic of GBM and a histopathological factor related to poor prognosis postoperatively. High Ki-67 rate can also signify high recurrence rate, the time between the first and second surgical resection and low survival rate (3). A study by Ozgiray et al. showed that Ki67 index changed between 12 and 34\% (11). Although suitable chemotherapy and radiotherapy were administered to patients postoperatively, they still experienced recurrence and metastasis that can be related to high Ki67 proliferation index (15). According to World Health 
Organization's new classification of central nervous system tumors in 2016, mutation in IDH-1 and IDH-2 (lesser) enzymes is found $10-15 \%$ of all GBMs. This significantly increases general survival rates, independently from prognostic factors, and although spinal metastasis is not related with mutation in adult GBMs, agents targeting this enzyme might play an important role for the treatment in the near future $(9,14)$.

Routine prophylactic craniospinal axis irradiation is not indicated for infratentorial GBM. Because nearly all recurrences are intracranial, spinal metastasis is rare and the prognosis is very poor. Nowadays, spinal GBM is treated with radiotherapy combined with surgery and most of the time followed by additional chemotherapy. Although many researchers believe that radiotherapy increases the survival rate in malignant spinal tumors (1), many others failed to show the relationship between radiotherapy and prognosis (5). Shirato et al. reported a 58-week survival in a case administered with a total radiation dose of 65 Gy (20). In this study, 2.5 Gy radiation dose was administered 4 times a week in a total of 5 weeks, making a total radiotherapy dose of 50 Gy. Our patients' survival period was between 4 and 12 weeks.

The need for chemotherapy in patients with spinal GBM remains controversial. Temozolomide and bevacizumab treatment concurrently administered with radiotherapy can change the recurrence pattern of the disease in areas that do not get direct treatment (19). Kaley et al. reported that temozolomide and bevacizumab have positive effects on survival (4). Temozolomide is accepted as an effective treatment for intracranial GBM, and it is recommended for patients with spinal GBM concurrently with radiotherapy and after radiotherapy in different doses (22). Therefore, concurrent radiotherapy and temozolomide were administered to our patients postoperatively, and temozolomide treatment was continued even after the radiotherapy. In spite of the concurrent temozolomide and radiotherapy treatment provided, the female patient developed metastasis in a short-term at 1 month both in cerebral regions that received radiotherapy and in the cervical and thoracic regions that did not receive radiotherapy.

Therefore, spinal metastases need further investigation in order to determine the best treatment (16). Indeed, spinal metastatis is associated with shorter survival than the nonspinal group, as shown in some studies (18). A subset of GBM patients benefited from the spinal screening, symptomatic spinal metastasis was detected early. In this study, symtomatic patients with higher $\mathrm{Ki}-67$ rate with wild-type IDH may be the predictive factor for spinal screening.

\section{CONCLUSION}

This study especially emphasizes the spinal metastasis and importance of spinal scan in patients with GBM. The immunohistochemical examination of intracranial GBM has been found to be effective in identifying the Ki-67 proliferation index rate in its spinal metastasis and recurrence. Spinal metastasis occurring in early stages of the disease is an indicator of poor prognosis and may shorten the survival period. Moreover, the new classification by WHO in 2016 indicated that although the relationship between IDH1 enzyme mutation and spinal metastases in adult patients with GBM is not clearly stated, pediatric cases of spinal cord and thalamus glioma are classified as "midline diffuse H3 K27M-mutant gliomas" if they carry histone H3 K27-mutation. The types with immunochemically high proliferation index (Ki-67) and IDH wild-types with poor prognostic features according to the new classification tend to be more metastatic in earlier stages of the disease; therefore, early-term spinal scans and radiotherapy over the spinal region might increase the survival rate.

This study revealed the following findings: Metastasis occurs more frequently in IDH-negative GBM than that in IDH-positive or NOS value. This indicated that MR imaging examination should be performed to administer a more efficient treatment.

Therefore, further and more extensive researches are warranted to confirm the findings of this study and to increase the survival rate.

\section{REFERENCES}

1. Adams H, Avendano J, Raza SM, Gokaslan ZL, Jallo GI, Quinones-Hinojosa A: Prognostic factors and survival in primary malignant astrocytomas of the spinal cord: A population-based analysis from 1973 to 2007 . Spine (Phila Pa 1976) 37:E727-E735, 2012

2. Birbilis TA, Matis GK, Eleftheriadis SG, Theodoropoulou EN, Sivridis E: Spinal metastasis of glioblastoma multiforme: An uncommon suspect? Spine (Phila Pa 1976) 35:e264-e269, 2010

3. Chen WJ, He DS, Tang RX, Ren FH, Chen G: Ki-67 is a valuable prognostic factor in gliomas: Evidence from a systematic review and meta-analysis. Asian Pac J cancer Prev 16:411420, 2015

4. Kaley TJ, Mondesire-Crump I, Gavrilovic IT: Temozolomide or bevacizumab for spinal cord high-grade gliomas. J Neurooncol 109:385-389, 2012

5. Lam S, Lin Y, Melkonian S: Analysis of risk factors and survival in pediatric high-grade spinal cord astrocytoma: A populationbased study. Pediatr Neurosurg 48:299-305, 2012

6. Lasocki A, Gaillard F, Tacey M, Durummond K, Stuckey S: Multifocal and multicentric glioblastoma: Improved characterisation with FLAIR imaging and prognostic implications. J Clin Neurosci 31:92-98, 2016

7. Lawton CD, Nagasawa DT, Yang I, Fessler RG, Smith ZA: Leptomeningeal spinal metastases from glioblastoma multiforme: Treatment and management of an uncommon manifestation of disease. J Neurosurg Spine 17:438-448, 2012

8. Louis DN, Ohgaki H, Wiestler OD, Cavenee WK, Burger PC, Jouvet A, Scheithauer BW, Kleihues P: The 2007 WHO classification of tumours of the central nervous system. Acta Neuropathol 114:97-109, 2007

9. Louis DN, Perry A, Reifenberger G, Von Deimling A, FigarellaBrander D, Cavenee WK, Ohgaki H, Wiestler OD, Kleihues P, Ellison DW: The 2016 World Health Organization Classification of Tumours of the Central Nervous System: A summary. Acta Neuropathol 131:803-820, 2016 
10. Onda K, Tanaka R, Takahashi H, Takeda N, Ikuta F: Cerebral glioblastoma with cerebrospinal fluid dissemination: A clinicopathological study of 14 cases examined by complete autopsy. Neurosurgery 25:533-540, 1989

11. Ozgiray E, Akay A, Ertan Y, Ertan Y, Cagli S, Oktar N, Ozdamar $\mathrm{N}$ : Primary glioblastoma of the medulla spinalis: A report of three cases and review of the literature. Turk Neurosurg 23:828-834, 2013

12. Ravindran K, Gaillard F, Lasocki A: Distant spread of a supratentorial glioblastoma to the spinal cord. J Clin Neurosci 38:56-57, 2017

13. Saito $R$, Kumabe $T$, Jokura $H$, Shirane $R$, Yoshimoto $T$ : Symptomatic spinal dissemination of malignant astrocytoma. J Neurooncol 61:227-235, 2003

14. Sanson M, Marie Y, Paris S, Idbaih A, Lafftaire J, Ducray F, El Hallani S, Boisselier B, Mokhtari K, Hoang-Xuan K, Delattre JY: Isocitrate dehydrogenase 1 codon 132 mutation is an important prognostic biomarker in gliomas. J Clin Oncol 27:4150-4154, 2009

15. Santi M, Mena H, Wong K, Koeller K, Olsen C, Rushing EJ: Spinal cord malignant astrocytomas. Clinicopathologic features in 36 cases. Cancer 98:554-561, 2003

16. Schindler G, Capper D, Korshunov A, Schmieder K, Brenke C: Spinal metastasis of gliosarcoma: Array-based comparative genomic hybridization for confirmation of metastatic spread. J Clin Neurosci 21:1945-1950, 2014

17. Shah A, Redhu R, Nadkarni T, Goel A: Supratentorial glioblastoma multiforme with spinal metastases. J Craniovertebr Junction Spine 1:126-129, 2010
18. Shibahara I, Saito R, Osada Y, Kanomori M, Sonada Y, Kumabe T, Mugikura S, Watanabe M, Tominaga T: Incidence of initial spinal metastasis in glioblastoma patients and the importance of spinal screening using MRI. J Neurooncol 141:337-345, 2019

19. Shields LB, Kadner R, Vitaz TW, Spalding AC: Concurrent bevacizumab and temozolomide alter the patterns of failure in radiation treatment of glioblastoma multiforme. Radiat Oncol 8:101, 2013

20. Shirato H, Kamada T, Hida K, Koyanagi I, Iwasaki Y, Miyasaka $\mathrm{K}$, Abe $\mathrm{H}$ : The role of radiotherapy in the management of spinal cord glioma. Int J Radiat Oncol Biol Phys 33:323-328, 1995

21. Stark AM, Nabavi A, Mehdorn HM, Blomer U: Glioblastoma multiforme-report of 267 cases treated at a single institution. Surg Neurol 63:162-169, 2005

22. Stupp R, Mason WP, van den Bent MJ, Weller M, Fisher B, Taphoorn MJ, Belanger K, Brandess AA, Marosi C, Bogdahn U, Curschmann J, Janzer RC, Ludwin SK, Gorlia T, Algeier A: Radiotherapy plus concomitant and adjuvant temozolomide for glioblastoma. N Engl J Med 352:987-996, 2005

23. Tinchon A, Oberndorfer S, Marosi C, Ruda R, Sax C, Calabek B, Grisold W: Malignant spinal cord compression in cerebral glioblastoma multiforme: A multicenter case series and review of the literature. J Neurooncol 110:221-226, 2012

24. Vertosick FT Jr, Selker RG: Brain stem and spinal metastases of supratentorial glioblastoma multiforme: A clinical series. Neurosurgery 27:516-521, 1990

25. Woesler B, Kuwert T, Probst-Cousin S, Schafers M, Wassmann H, Gullota F, Schober O: Spinal metastases of a high-grade astrocytoma visualized with FDG-PET. Clin Nucl Med 22:863864, 1997 